\title{
Aplicação do checklist para cirurgia segura: Relato de experiência
}

\author{
Applying the safe surgery checklist: An experience report \\ Aplicación del checklist de cirugía segura: Relato de experiencia
}

\author{
Ana Paula Pancieri ${ }^{1}$, Rachel de Carvalho ${ }^{2}$, Eliana Mara Braga ${ }^{3}$
}

RESUMO: Objetivo: relatar a experiência da aplicação do checklist de cirurgia segura proposto pela OMS. Método: pesquisa descritiva, narrativa, de nível I, do tipo relato de experiência com a aplicação do checklist de cirurgia segura em 30 procedimentos anestésicocirúrgicos realizados em um hospital escola, situado no interior do Estado de São Paulo, no segundo semestre de 2011, seguindo-se as três etapas preconizadas pela OMS. Conclusão: identificou-se a necessidade de inclusão e mudança de alguns itens do checklist e preenchimento de itens na sala de recuperação pós-anestésica. É importante o enfermeiro e o coordenador da lista terem domínio de como realizar a checagem e saber conduzir com responsabilidade e ética todas as etapas propostas, além de enfatizar a responsabilidade de cada profissional participante. A comunicação é essencial para o bom andamento do procedimento e o checklist faz com que isso ocorra da melhor maneira possível.

PALAVRAS-CHAVE: Segurança do paciente. Salas cirúrgicas. Lista de checagem. Enfermagem de centro cirúrgico. Enfermagem perioperatória.

ABSTRACT: Objective: to report the experience in applying the safe surgery checklist proposed by the World Health Organization (WHO). Method: a descriptive, narrative, level 1 study, of experience report type, with the application of the safe surgery checklist, following the three stages recommended by WHO, to 30 anesthetic and surgical procedures carried out in a teaching hospital in São Paulo state in the second semester of 2011. Conclusion: we identified the need to include and change some items in the checklist and fill in the items in the post-anesthetic recovery room. It's important that the nurse or the checklist coordinator are able to master the checking, know how to conduct all the proposed items responsibly and ethically, and emphasize the responsibility of each participant professional. Communication is essential for the proper conduct of this procedure and the checklist guarantees its best performance.

KEYWORDS: Patient safety. Operating rooms. Checklist. Operating room nursing. Perioperative nursing.

RESUMEN: Objetivo: relatar la experiencia de aplicación del checklist de cirugía segura propuesta por la OMS. Método: estudio descriptivo, narrativo, de nivel 1, del tipo relato de experiencia, con la aplicación del checklist de cirugía segura en 30 procedimientos anestésico-quirúrgicos, realizados en un hospital universitario, ubicado en el interior del Estado de São Paulo, durante el segundo semestre de 2011, siguiendo las tres etapas recomendadas por la OMS. Conclusión: se identificó la necesidad de incluir y cambiar algunos ítems del checklist, y rellenarlos en la sala de recuperación. Es importante que el enfermero y el coordinador de la lista sepan realizar el chequeo y conduzcan con responsabilidad y ética todas las etapas propuestas, además de enfatizar la responsabilidad de cada uno de los profesionales participantes. La comunicación es esencial para la buena ejecución del procedimiento y el checklist hace que eso ocurra de la mejor manera posible.

PALABRAS ClAVE: Seguridad del paciente. Quirófanos. Lista de chequeo. Enfermería de quirófano. Enfermería perioperativa.

${ }^{1}$ Enfermeira. Especialista em Centro Cirúrgico, Recuperação Anestésica e Centro de Material e Esterilização pela Faculdade de Enfermagem do Hospital Israelita Albert Einstein (FEHIAE). Enfermeira no Hospital das Clínicas da Faculdade de Medicina de Botucatu (HCFMB).

Rua dos Operários, 373. Centro. CEP 13710-000. Tambaú, SP, Brasil.

Telefone: (19) 99776-2983. E-mail: appancieri@yahoo.com.br

${ }^{2}$ Enfermeira. Especialista em Cardiologia e Centro Cirúrgico. Mestre e Doutora em Enfermagem pela Escola de Enfermagem da USP. Docente dos Cursos de Graduação e de Pós-Graduação da FEHIAE. Coordenadora do Curso de Pós-Graduação da FEHIAE. E-mail: rachel.carvalho@einstein.br

${ }^{3}$ Enfermeira. Professora Doutora do Departamento de Enfermagem da Faculdade de Medicina de Botucatu (UNESP). Botucatu, SP, Brasil. E-mail: elmara@ @mb.unesp.br 


\section{Introdução}

Em 2008, a Organização Mundial de Saúde (OMS) lançou a campanha 'Cirurgias Seguras Salvam Vidas', que pretende reduzir a ocorrência de danos ao paciente cirúrgico e definir padrões de segurança que podem ser aplicados a todos os países membros da $\mathrm{OMS}^{1}$. No mesmo ano, dados coletados pela OMS mostraram o número total de 234 milhões de cirurgias pelo mundo, sendo que cerca de sete milhões de pessoas enfrentaram complicações provenientes de cirurgias ${ }^{1}$.

Considera-se inadmissível não utilizar todo o conhecimento adquirido com a evolução técnico-científica para prevenir complicações, iatrogenias e eventos adversos. Isso levou a OMS e a Universidade de Harvard a iniciarem a campanha para realização de cirurgias seguras, preparando como modelo um checklist composto por três partes²:

- Identificação ou Sign in (antes da indução anestésica): quando se verifica verbalmente a identidade do paciente, o procedimento e o local da cirurgia, e se o consentimento para o procedimento foi assinado. O coordenador da lista observa se o lado correto da cirurgia foi sinalizado e confere se o oxímetro de pulso foi colocado corretamente no paciente e está funcionando. Deve rever verbalmente, com a equipe de anestesia, se o paciente possui vias aéreas de difícil acesso, risco de perda sanguínea ou de reação alérgica, de modo a garantir a segurança durante o procedimento anestésico. O ideal seria que o cirurgião estivesse presente nesta fase, já que este pode ter uma ideia mais clara sobre os fatores complicadores; contudo, a presença do cirurgião não é essencial para completar essa primeira parte do checklist;

- Confirmação ou Timeout (antes da incisão na pele - pausa cirúrgica): todos os profissionais presentes na sala de operações e que irão participar ativamente do procedimento se apresentam (nome e função); faz-se a conferência, em voz alta, da identidade do paciente, do procedimento e da parte do corpo que será operada. Em seguida, o cirurgião, o anestesiologista e o membro da equipe de enfermagem, verbalmente, revisam os pontos críticos para a cirurgia, fazendo uso do checklist e confirmando o uso profilático de antibióticos nos últimos 60 minutos; além disso, certificam-se da disponibilidade dos exames de imagem;

- Registro ou Sign out (antes do paciente sair da sala cirúrgica): em conjunto com a equipe, o coordenador da lista analisa o procedimento, contam-se as compressas e os instrumentos, rotulam-se as peças anatômicas ou outras amostras obtidas, checam-se informações sobre quaisquer danos nos equipamentos, assim como outros problemas a serem resolvidos; finalizam traçando os planos de cuidados em relação ao pós-operatório, antes do encaminhamento do paciente à sala de recuperação anestésica ${ }^{1}$

Simples cuidados antes do procedimento cirúrgico podem impedir complicações para o paciente, fazendo a diferença entre o sucesso e o fracasso da anestesia e da cirurgia ${ }^{2}$.
São muitos os fatores que podem levar uma equipe cirúrgica ao erro, colocando em risco a segurança do paciente. Entre esses fatores, podem-se citar: materiais inadequados, por esterilização inadequada ou por mau funcionamento; corpo estranho esquecido no paciente, como instrumentais e compressas; dificuldade em reconhecer complicações durante a cirurgia; planejamento inadequado dos cuidados no período pós-operatório; perfurações ou hemorragias; intervenção com tempo prolongado e cirurgias de sítio e/ou indivíduo errados, ou, ainda, procedimento errado. Quase metade dos sujeitos de um estudo realizado na Guatemala relatou que já cometeram algum erro que poderia ter sido prevenido ${ }^{3}$.

Existem outras situações que acabam passando despercebidas, por serem corriqueiras, sendo, por este motivo, de difícil mensuração, como a sobrecarga de trabalho - gerada pelas longas jornadas ou pela execução de diferentes tarefas concomitantes - e as interrupções constantes no procedimento, além da troca de pessoal: todos estes também são fatores que contribuem para a ocorrência de erros ${ }^{4,5}$.

A avaliação do uso do checklist em oito instituições-piloto no mundo mostrou que sua aplicação praticamente dobrou as chances de os usuários receberem o tratamento cirúrgico com padrões de cuidado adequados ${ }^{4,6}$. Não se sabe o mecanismo responsável por esse evento, mas acredita-se que se deva a mudanças na rotina, no comportamento da equipe, de cada membro individual e coletivamente, e na comunicação interpessoal ${ }^{5,7}$.

Os objetivos da OMS com a campanha Cirurgia Segura incluem diminuir a morbimortalidade de pacientes cirúrgicos, dando, às equipes cirúrgicas e aos administradores hospitalares, orientações sobre a função de cada indivíduo e qual é o padrão de uma cirurgia segura, além de oferecer um instrumento de avaliação uniforme do serviço para vigilância nacional e internacional. As normas a serem seguidas podem ser utilizadas em qualquer parte do mundo e devem ser adaptadas de acordo com a realidade institucional ${ }^{1}$.

A implementação do checklist é de baixo custo. Estimase que seja necessário o tempo médio de três minutos para aplicação das três fases do processo de verificação e orienta-se que um único profissional, que participa do procedimento cirúrgico, seja responsável por essa aplicação, que é chamado de coordenador da lista ${ }^{1}$. Esse profissional deve ter conhecimento sobre o processo anestésico-cirúrgico, estando apto a interromper o procedimento ou impedir seu avanço, se julgar insatisfatório um item necessário ${ }^{8}$.

A introdução do checklist é um passo para uma nova cultura de segurança na sala cirúrgica ${ }^{7}$. Realizar a checagem por meio do coordenador, com participação do paciente e da equipe multiprofissional, é essencial para o sucesso do procedimento ${ }^{7}$. Quando há trabalho coletivo, os integrantes da equipe passam a se perceber mais do que meros executores de tarefas, resgatando a dimensão afetiva do trabalho ${ }^{9}$.

Diante das considerações realizadas e da importância da criação e da aplicação do checklist para a segurança do 
paciente, para a equipe cirúrgica e para a própria instituição, surgiu a motivação para realizar a presente pesquisa.

\section{Objetivo}

Relatar a experiência da aplicação do checklist de cirurgia segura proposto pela Organização Mundial de Saúde em 30 procedimentos anestésico-cirúrgicos, realizados em um hospital escola do interior do Estado de São Paulo.

\section{Método}

Trata-se de uma pesquisa descritiva, narrativa, de nível I, do tipo relato de experiência, que tem a finalidade de descrever o trabalho desenvolvido pela primeira autora em um hospital escola situado no interior do Estado de São Paulo.

$\mathrm{O}$ estudo tem o intuito de demonstrar como foi realizada a aplicação do checklist proposto pela $\mathrm{OMS}^{1}$ (Anexo A) em 30 procedimentos anestésico-cirúrgicos e as dificuldades encontradas na aplicação deste processo, bem como a percepção da autora acerca da opinião da equipe cirúrgica sobre o checklist.

Descreve-se a padronização do impresso utilizado, a aplicação do mesmo nos procedimentos e as dificuldades que foram encontradas no processo, bem como as alterações realizadas no checklist proposto pela OMS para uso na instituição, a fim de que se possa estudar a continuidade da aplicação do checklist em todos os procedimentos realizados no Centro Cirúrgico (CC) da instituição, objetivando, desta forma, a eficácia da assistência prestada e a segurança do paciente.

A experiência relatada trata-se de um estudo realizado no CC de um hospital escola em uma instituição pública no interior do Estado de São Paulo, no segundo semestre de 2011. O CC da instituição estudada é constituído por 16 salas operatórias, com 11 em funcionamento no período do estudo. Atende cirurgias de pequeno, médio e grande porte de 12 especialidades (Cirurgia Cardíaca, Cirurgia Pediátrica, Cirurgia Torácica, Cirurgia Vascular, Gastrocirurgia, Neurocirurgia, Oftalmologia, Otorrinolaringologia, Ortopedia, Ginecologia, Cirurgia Plástica e Urologia), de acordo com o horário semanal estabelecido para cada equipe. Realiza aproximadamente 9.000 cirurgias/ano. Para ser realizado, o estudo precisou da autorização do docente responsável por cada disciplina, pelo responsável pela disciplina de Anestesiologia e pelos responsáveis pela unidade de CC, para ser aprovado pelo Comitê de Ética em Pesquisa da Instituição. Foi autorizado pelos responsáveis da unidade, pelo docente da Anestesiologia e por oito das 12 especialidades cirúrgicas, além do Comitê de Ética em Pesquisa, mediante ofício 130/11.

\section{Resultados e Discussão}

O relato de experiência foi baseado na aplicação do checklist de cirurgia segura proposto pela OMS em 30 procedimentos anestésico-cirúrgicos de oito especialidades cirúrgicas de um hospital escola. Optou-se pelo modelo proposto para identificar modificações necessárias para a unidade.

Durante a experiência, a pesquisadora atuou na unidade de CC, aplicando o checklist, acompanhando procedimentos anestésico-cirúrgicos, prestando assistência ao paciente no período transoperatório, preparando a sala e provendo materiais e equipamentos necessários para cada procedimento, encaminhando materiais para a central de material e esterilização, dentre outras atividades fora da área assistencial, como realização de escala diária e mensal de funcionários, montagem do mapa cirúrgico diário e programa de educação continuada com treinamento para os funcionários da unidade para aplicação do checklist.

A aplicação do checklist seguiu o modelo proposto pela OMS. Na chegada do paciente ao CC, era checado, com ele ou seu acompanhante, a identidade do paciente, o sítio cirúrgico, o procedimento proposto e o termo de consentimento corretamente preenchido e devidamente assinado. Durante a identificação, apenas uma vez o responsável pelo paciente relatou não ter ciência do motivo da realização do procedimento cirúrgico. No mesmo momento, foi solicitado ao cirurgião que fosse esclarecer as dúvidas, o que foi atendido prontamente.

Em seguida, encaminhava-se o paciente à recuperação anestésica para aguardar o término do preparo da sala ou diretamente para a sala cirúrgica, caso esta já estivesse pronta.

\section{Sign in}

Quando o paciente já estava na sala, antes de iniciar a anestesia, terminava-se de checar a primeira parte do checklist (Identificação ou Sign in). Mais uma vez, confirmava a identificação e o procedimento a ser realizado;na sequência, se a verificação anestésica estava concluída e o oxímetro funcionando; se o paciente possuía alguma alergia conhecida, via aérea difícil, risco para aspiração e risco para perda sanguínea, com necessidade de acesso adequado para infusão de fluidos ou hemocomponentes.

No momento antes da indução anestésica, não foram observados problemas ou dificuldades na troca de informações entre os profissionais. Os itens checados já faziam parte da prática local, porém não confirmados entre toda a equipe e nem anotados, apenas confirmados verbalmente entre os médicos. Apesar de não verificar problemas nas cirurgias em que o checklist foi aplicado, no mesmo período observou-se a necessidade de acrescentar alguns outros itens a esta etapa, como: ambu testado, foco funcionando e posicionamento da mesa cirúrgica conferido. 


\section{Timeout}

Ao final da indução anestésica, auxiliava-se o posicionamento do paciente e a paramentação da equipe cirúrgica. Antes da realização da incisão cirúrgica, era feita a segunda parte do checklist (Confirmação ou Timeout). Confirmava-se se toda a equipe estava presente na sala cirúrgica e se todos se conheciam pelo nome e função. A resposta era sempre positiva, pois, ao chegar à sala, se houvesse alguém desconhecido, já era feita a sua apresentação entre os profissionais.

Confirmava-se novamente o nome do paciente, o procedimento proposto e o sítio cirúrgico; então, partia-se para a revisão do cirurgião sobre etapas críticas previstas. Verificou-se maior dificuldade entre os médicos residentes mais novos, especialmente em verbalizar esta informação.

Alguns dos eventos mais relatados foram sangramento inesperado e morte súbita do paciente. Quando o sangramento era previsto, discutia-se sobre os hemocomponentes solicitados ao banco de sangue. Não houve problemas quanto a este aspecto, mas se identificou a necessidade de deixar anotado no checklist quais hemocomponentes foram solicitados. Na revisão da parte de anestesia, as preocupações eram muitas vezes as mesmas do cirurgião e, na revisão da parte de enfermagem sobre materiais e equipamentos, não houve problemas em nenhum dos 30 procedimentos nos quais o checklist foi aplicado.

A seguir, era questionado sobre a profilaxia antimicrobiana. Foi nesta etapa que houve necessidade de mais interrupções no procedimento. Algumas vezes, a profilaxia ainda não havia sido realizada, pois os médicos deixavam a receita para que o circulante buscasse a medicação e, ao atender as necessidades da sala, isso ia ficando para depois. Ao acontecerem situações dessa maneira, foi solicitado que se esperasse até ser feita a aplicação do antibiótico e, em nenhum procedimento em que isso ocorreu, houve desentendimentos. A equipe aguardou até o momento da administração do medicamento para iniciar a cirurgia. Também ocorreu que a equipe cirúrgica optou pela não realização da antibioticoprofilaxia em sala operatória, pois o paciente já estava em uso de antimicrobiano; porém, ao checar a prescrição, o anestesiologista verificou que esta fora realizada havia mais de uma hora, necessitando de outra dose. Também foi aguardado até o momento da administração do antimicrobiano para iniciar a incisão cirúrgica. Diante destas situações, optou-se por acrescentar, na etapa de profilaxia antimicrobiana, o horário e o nome do medicamento administrado.

Terminava-se esta etapa questionando sobre exames de imagem presentes na sala e, em nenhum dos procedimentos, houve interrupções devido a este item. Após a confirmação, a equipe iniciava o procedimento cirúrgico.

\section{Sign out}

Finalizado o procedimento, checava-se a contagem de instrumentais, compressas e agulhas. Nos 30 procedimentos acompanhados, a contagem sempre estava correta. A seguir, era verificado se havia amostras para anatomia, e, caso houvesse, checava se estavam corretamente identificadas. Funcionários da unidade relataram que algumas vezes os médicos se esqueciam de preencher o pedido da amostra para envio ao laboratório. Então, optou-se por acrescentar, neste item, qual era a amostra e se o pedido foi preenchido corretamente pelo médico.

Problemas com equipamentos ou instrumentais durante o procedimento também eram checados. Quando houve, os equipamentos ou instrumentais eram identificados com a informação 'reclamação de não funcionamento' e comunicava-se à enfermeira responsável sobre o problema identificado, a fim de que fossem tomadas as devidas providências.

Optamos, durante a experiência nos primeiros procedimentos, por checar o item 'registro do procedimento' na sala de recuperação anestésica e não na sala cirúrgica, a fim de evitar atrasos na limpeza da sala e o início da cirurgia seguinte. Então, optamos por realizar mais uma etapa ('Antes de o paciente deixar a recuperação anestésica'), uma vez que isso já era de praxe, pois, em se tratando de um hospital escola, as cirurgias da especialidade do dia eram realizadas pela mesma equipe, que aproveitava o intervalo de limpeza de sala entre uma cirurgia e outra para anotar o procedimento. A verificação e a entrega da folha de registro do procedimento eram feitas à equipe da recuperação, para ser anexada ao procedimento.

E, por último, antes de o paciente sair da sala cirúrgica, realizava-se a checagem do último item, com as devidas recomendações para a recuperação. Quanto a este planejamento, observou-se a mesma dificuldade que a encontrada nas etapas críticas, por parte dos médicos. Alguns não sabiam como expressar isso com a equipe da sala cirúrgica, talvez por falta de hábito. A expressão 'cuidados essenciais para o transporte e a recuperação do paciente' também gerou divergências de entendimento. Alguns médicos citaram os cuidados necessários para o paciente até a sala de recuperação anestésica, enquanto outros traçaram um plano de cuidados até a alta hospitalar. Essa etapa necessita de maturidade e experiência do enfermeiro para intervir e, em conjunto com toda a equipe, traçar o melhor plano de cuidados para a recuperação pós-anestésica e pós-operatória do paciente.

Observou-se, também, a dificuldade da equipe de enfermagem em entender a importância da realização da checagem nos momentos preconizados pela OMS e, principalmente, com a presença de todos. Baseando-se apenas na experiência da aplicação do checklist nesta unidade, propôs-se uma lista com as alterações julgadas necessárias, para ser aplicada na Instituição sede do estudo (Anexo B).

Todas as cirurgias do referido hospital são realizadas por cirurgiões e por médicos residentes em cirurgia. Durante a experiência, observou-se que muitos não conheciam o checklist proposto pela OMS, enquanto outros já o tinham 
como aplicativo em dispositivo móvel pessoal, porém, sem utilizá-lo, diferentemente da equipe de enfermagem, na qual poucos não conheciam o checklist de cirurgia segura.

Quando analisamos outro estudo, verificamos que a maioria dos sujeitos já conhecia o checklist, o que torna preocupante o fato de alguns profissionais do estudo relatado não o conhecerem. Porém, ao mesmo tempo em que tinham conhecimento, alguns não estavam cientes dos objetivos do checklist e um número ainda maior não sabia que este é aplicado em três etapas ${ }^{3}$.

$O$ fato de a equipe de enfermagem conhecer o checklist não significa saber utilizá-lo corretamente. Realizar treinamentos com todos os profissionais que irão atuar na sala operatória é imprescindível para o sucesso do programa de cirurgia segura. Utilizar o checklist é muito mais do que simplesmente checar uma lista. Enquanto não for mostrado a todos o porquê e como utilizá-lo corretamente, a equipe não estará preparada para fazer seu uso ${ }^{10}$.

É necessário desfazer o estigma de que esta checagem é uma imposição por parte do enfermeiro, pois o grupo todo deve colaborar para a segurança do paciente.

Aplicar o checklist de cirurgia segura é muito mais do que apenas dizer 'Ei, pessoal, é isso que eu quero fazer e preciso de apoio'. Os responsáveis pelo CC devem adotar essa ferramenta para complementar a segurança do paciente e necessitam conscientizar sua equipe sobre a importância de usá-la adequadamente, pois, se eles não entenderem o porquê de realizar a checagem corretamente, eles não estarão preparados para sua aplicação ${ }^{10}$.

Assim como na experiência relatada, observaram-se quase erros na profilaxia antimicrobiana. Outro estudo mostra que o checklist incentiva o uso de antibióticos na sala operatória, o que mostrou uma diminuição nas taxas de infecção de sítio cirúrgico ${ }^{6}$.

$\mathrm{O}$ checklist não é apenas uma ferramenta para garantir segurança ao paciente, mas é, também, um importante método de melhorar a comunicação na sala cirúrgica ${ }^{11-13}$. Ele nos dá a oportunidade de expressar as preocupações a todos os membros da equipe cirúrgica. Entretanto, a comunicação na sala é algo que ainda precisa ser melhorado, uma vez que muitos profissionais têm dificuldade em compartilhar informações verbalmente ${ }^{14,15}$. A verbalização e a previsão do procedimento cirúrgico, realizadas repetidas vezes pelos membros da equipe cirúrgica, provavelmente melhoram o desempenho da equipe ${ }^{5}$.

Assim como identificado, a dificuldade na compreensão de alguns itens do checklist não foi apenas no hospital onde o estudo foi realizado. A experiência de aplicação, como este estudo, é importante para identificar as dificuldades da equipe e, então, poder sugerir melhorias na lista. Realizar treinamentos e estruturar o checklist com uma linguagem simples é imprescindível, ao mesmo tempo em que é interessante incluí-lo nos impressos já existentes para evitar documentos duplicados ${ }^{16}$.
Os médicos que utilizam o checklist têm uma boa visão desta ferramenta, percebendo o valor do seu uso e relatando que gostariam que fosse usado se estivessem em um procedimento cirúrgico ${ }^{13}$.

\section{Considerações finais}

Aplicar o checklist requer do enfermeiro ou do coordenador da lista um conhecimento de como realizá-lo em todas as etapas. É necessário conseguir envolver toda a equipe durante a checagem, para que todos respeitem cada um dos itens da lista e tenham a consciência de que, para sua realização, é necessário fazer e não apenas fingir que se faz. Para isso, é preciso enfatizar a responsabilidade de cada profissional durante o procedimento anestésico-cirúrgico e a ética pela profissão. A comunicação é essencial para o bom andamento do procedimento e o checklist faz com que isso ocorra da melhor maneira possível.

Começar um procedimento com conhecimento das preocupações do cirurgião, do anestesiologista e da equipe de enfermagem faz com que qualquer intercorrência transcorra de maneira menos estressante. Rever os cuidados necessários para o pós-operatório é importante para que o paciente receba toda a assistência da melhor forma possível para sua recuperação.

Conhecer a rotina de outros hospitais que já utilizam o checklist é muito importante para que o desenvolvimento de uma lista, especialmente para a sua unidade, seja elaborada da melhor maneira e que seu uso e visualização sejam fáceis e eficientes.

\section{Referências}

1. Organização Mundial da Saúde - OMS. Segundo desafio global para a segurança do paciente. Cirurgias Seguras Salvam Vidas. Tradução Nilo MS, Duran IA. Rio de Janeiro: OPAS; 2009.

2. Ferraz EM. A cirurgia segura: uma exigência do século XXI. Rev Col Bras Cir. 2009;36(4):281-2. PMid:20076914. http://dx.doi. org/10.1590/S0100-69912009000400001

3. Hurtado JJ, Jimenez X, Peñalonzo MA, Villatoro C, Izquierdo S, Cifuentes M. Acceptance of the WHO Surgical Safety Checklist among surgical personnel in hospitals in Guatemala city. BMC Health Serv Res. 2012;12:169. PMid:22721269 PMCid:PMC3444374. http://dx.doi.org/10.1186/1472-6963-12-169

4. Pedreira MLG, Harada MJCS. Enfermagem dia a dia: segurança do paciente. In: Salles CLS, Carrara D. Cirurgia segura. São Caetano do Sul: Yendis; 2009. p. 109-17.

5. Abdel-Rehim S, Morritt A, Perks G. WHO Surgical Checklist and its practical application in plastic surgery. Plast Surg Int 2011; [cited 2012 dec 01]. Available from: http://www.ncbi.nlm.nih.gov/ pmc/articles/PMC3335640/pdf/PSI2011-579579.pdf.

6. Haynes AB, Weiser TG, Berry WR, Lipsitz SR, Breizat AHS, Dellinger P, et al. A surgical safety checklist to reduce morbidity and mortality in a global population. N Engl J Med. 2009;360:4919. PMid:19144931. http://dx.doi.org/10.1056/NEJMsa0810119 
7. Schalack WS, Boermeester MA. Patient safety during anesthesia: incorporation of the WHO safe surgery guidelines into clinical practice. Curr Opin Anesthesiol. 2010;23:754-8. PMid:20930622. http://dx.doi.org/10.1097/ACO.0b013e3283400b26

8. World Health Organization - WHO. Checklists Save Lives. Bull World Health Organ. 2008;86(7):501-2. http://dx.doi.org/10.2471/ BLT.08.010708

9. Braga EM, Berti HW, Risso ACMCR, Silva MJP. Relações interpessoais da equipe de enfermagem em centro cirúrgico. Rev SOBECC. 2009;14(1):22-9.

10. Conley DM, Singer SJ, Edmondson L, Berry WR, Gawande AA. Effective surgical safety checklist implementation. J Am Coll Surg. 2011;212(5):873-9. PMid:21398154. http://dx.doi.org/10.1016/j. jamcollsurg.2011.01.052

11. Cunat C, Flatin V, Viale J-P. Stratégie de deployment de la checklist dans un CHU. Ann Fr Anesth Reanim. 2011;30(6):484-8. PMid:21620639. http://dx.doi.org/10.1016/j.annfar.2011.04.004

12. Helmiö P, Blomgren K, Takala A, Pauniaho SL, Takala RS, Ikoken TS. Towards better patient safety: WHO Surgical Safety Checklist in otorhinolaryngology. Clin Otolaryngol.
2011;36(3):242-7. PMid:21481197. http://dx.doi. org/10.1111/j.1749-4486.2011.02315.x

13. Haynes AB, Weiser TG, Berry WR, Lipsitz SR, Breizat AHS, Dellinger EP, et al. Changes in safety attitude and relationship to decreased postoperative morbidity and mortality following implementation of a checklist-based surgical safety intervention. BMJ Qual Saf. 2011;20:102-7. PMid:21228082. http://dx.doi. org/10.1136/bmjqs.2009.040022

14. Paugam-Burtz C, Guerrero O. Check-list sécurité au bloc opératoire: le bilan après un an de deployment à l'hôpital Beaujon. Ann Fr Anesth Reanim. 2011;30(6):475-8. PMid:21612886. http:// dx.doi.org/10.1016/j.annfar.2011.04.005

15. Rateau F, Levraut L, Colombel AL, Bernard JL, Quaranta JF, Cabarrot P, et al. Check-list "Sécurité du patient au bloc opératoire": une anée d'expérience su 40000 interventions au centre hospitalier de Nice. Ann Fr Anesth Reanim. 2011;30(6):479-83. PMid:21601412. http://dx.doi.org/10.1016/j.annfar.2011.04.003

16. Gueguen T, Coevoet V, Mougeot M, Pierron A, Blanquart D, Voicu M et al. Déploiement de la check-list "Sécurité du patient au bloc opératoire" dans deux hôpitaux lorrains. Performances et difficulties. Ann Fr Anesth Reanim. 2011;30(6):489-94. PMid:21616629. http://dx.doi.org/10.1016/j.annfar.2011.04.006

\section{-1 RUHOF}

\section{ENDOZIME BIO-CLEAN}

\author{
0 único detergente \\ enzimático capaz de \\ remover o biofilme, \\ conforme norma \\ ISO 15883-5 anexo F.
}
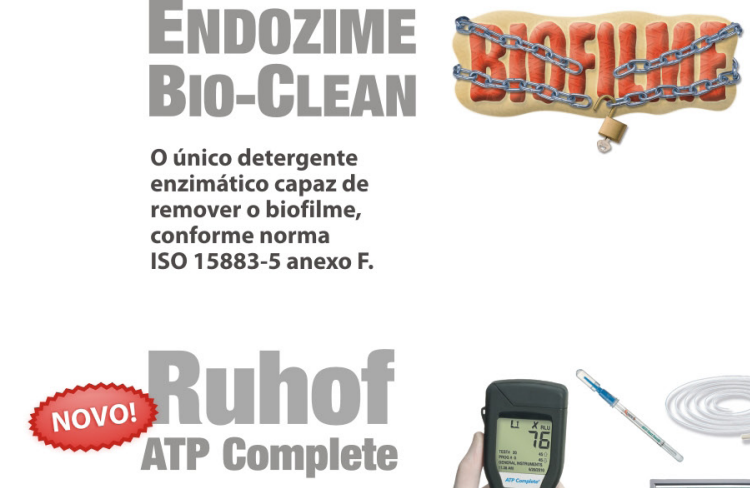

Novo software de armazenagem e rastreabilidade de dados.

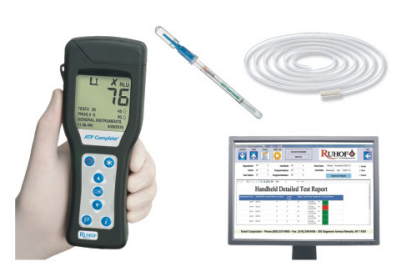

\section{SISTEMA DE EMBALAGENS PARA ESTERILIZAÇ̃̃O PARA ELIMINACÃO DE PACOTES MOLHADOS}

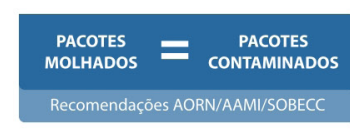

As embalagens para esterilização Cleantech são produzidas em Nãotecido SMS Grau Médico proporcionando um sistema de barreira estéril, em conformidade com a Norma ISO 11607.

Possuem dupla proteção:

$$
\begin{array}{r}
\text { embalagem } \\
\text { hidrofóbica } \\
\text { barreira microbiana } \\
\text { comprovada através } \\
\text { de laudos técnicos } \\
\text { embalagem } \\
\text { hidrofílica } \\
\text { eliminação dos } \\
\text { pacotes molhados } \\
\text { através da dissipação } \\
\text { de calor e umidade }
\end{array}
$$

\section{Cleantech}

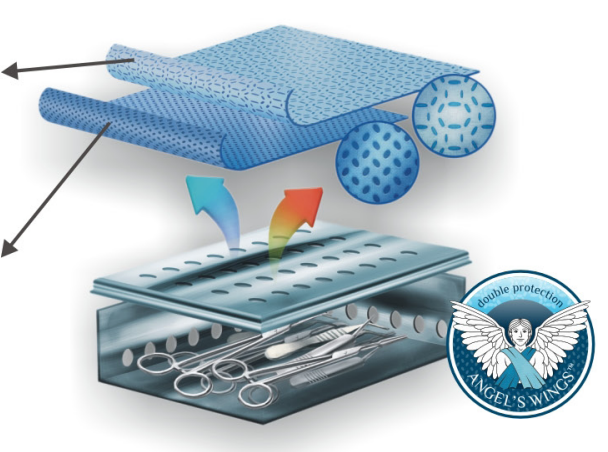

CPL a Mitrade

Porto Alegre/ RS | 5133586300

www.planitrade.com.br

planitrade@planitrade.com.br
- Maccleall

Porto Alegre/ RS | 5133754500

www.medclean.com.br

meddlean@medclean.com.br
SE VOCÊ NÃO TEVE OPORTUNIDADE DE PARTICIPAR DOS SIMPÓSIOS-SATÉLITES REALIZADOS NO CONGRESSO SOBECC, PODERÁ ASSISTIR AOS CONTEUDOS NA ÍNTEGRA, DISPONIBILIZADOS NO SITE www.planitrade.com.br

Desafios para Detergentes Enzimáticos: Registros, Qualificação e Validação de Limpeza (Ing/Port) Palestrante: Marc Esquenet - Químico-Chefe e Vice-Presidente de Pesquisa e Desenvolvimento da Ruhof Corporation

Como Aumentar a Produtividade do seu Bloco Cirúrgico? Novos Conceitos que Proporcionam Alto Giro de Sala e Práticas Cirúrgicas Seguras

Palestrante: Enfa Lea Pereira de Souza - Especializada em CC, RPA e CME pela USP e sócia proprietária da LEAH Treinamento e Orientação em Processos Hospitalares 
Anexo A. Checklist proposto pela OMS (2009) ${ }^{1}$.

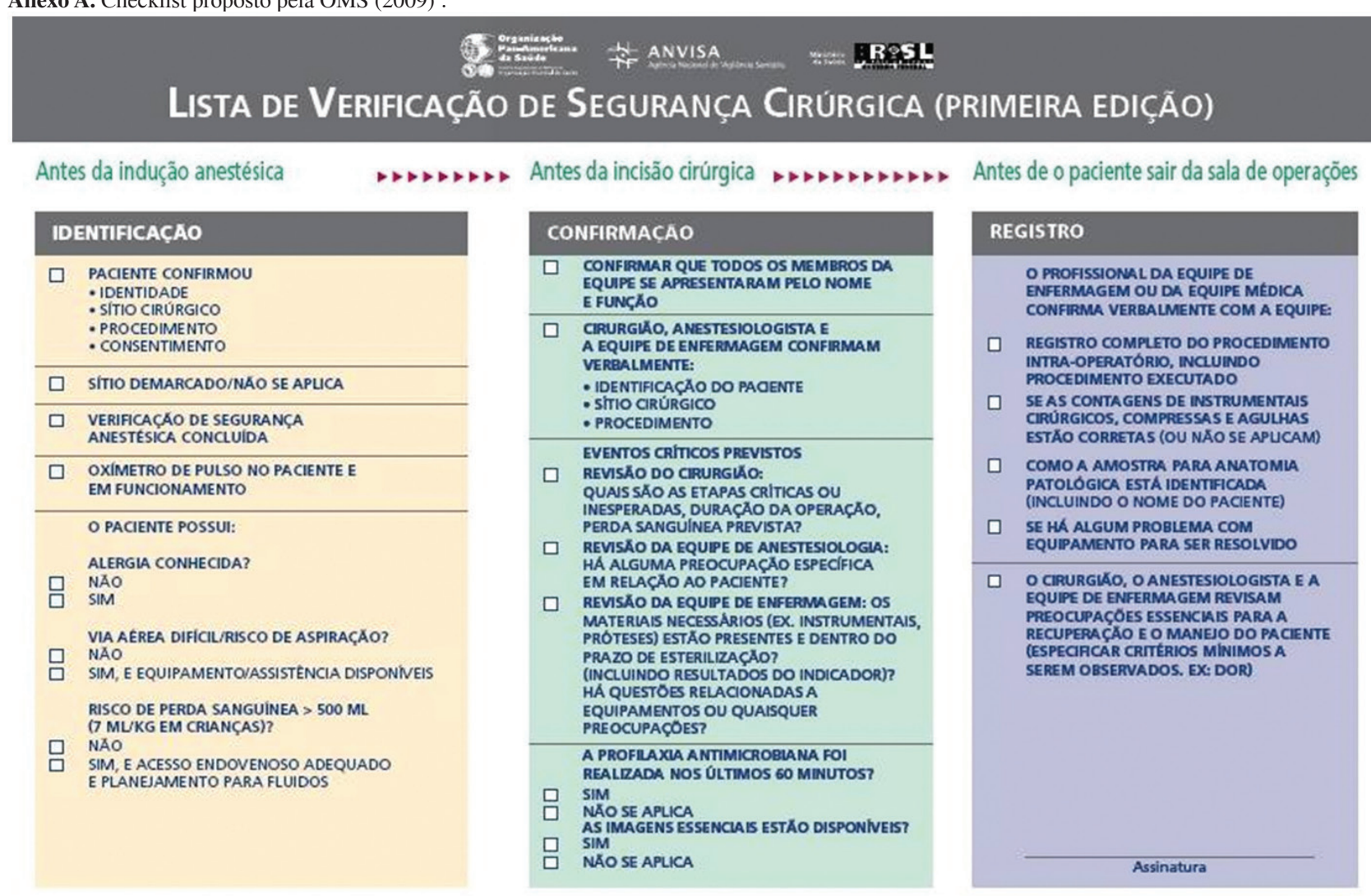

ESTA LISTA DE VERIFICAÇAO NAO TEM A INTENÇÃO DE SER ABRANGENTE. A CRESCIMOS E MODIFICAÇOES PARA ADAPTAÇAO A PRATICA LOCAL SAOO RECOMENDADOS.

Fonte: Organização Mundial da Saúde (OMS). Segundo desafio global para a segurança do paciente. Cirurgias Seguras Salvam Vidas. Tradução Nilo MS, Duran IA. Rio de Janeiro: Organização Panamericana da Saúde (OPAS); 2009. 
Anexo B. Checklist proposto para a unidade de Centro Cirúrgico na Instituição sede do estudo.

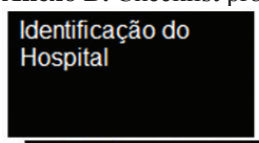

LISTA DE VERIFICAÇÃo DE SEGURANÇA CIRÚRGICA

\section{Identificação do} Hospital

\begin{tabular}{|l|l|}
\hline NOME DO PACIENTE_ & NEXO_OU ETIQUETA) \\
\hline AMBU TESTADO & () SIM () NÄO SE APLICA \\
FOCO FUNCIONANDO & () SIM () NÄO SE APLICA
\end{tabular}

ESPECIALIDADE PROCEDIMENTO

DATA SALA

) TODOS OS MEMBROS DA EQUIPE SE CONHECEM PELO NOME E FUNÇĀO?

POSICIONAMENTO DA MESA CIRÚRGICA () TESTADO

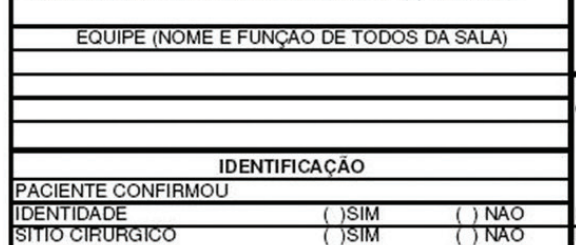

() TODOS CONFIRMAM VERBALMENTE:

IDENTIFICAÇĀO DO PACIENTE

SITIO CIRÚRGICO

PROCEDIMENTO

CIRURGIÃO

EVENTOS CRITICOS PREVISTOS

( ) ETAPAS CRÍTICAS/INESPERADAS?

SITIOCIRURG

PROCEDIMENTO

()SIM () NAOO

(ISIM

() NAO

SE APLICA

ANTES DA INDUCAO ANESTESICA

(1) VERIFICACÃO DE SEGURANCA ANESTÉSICA CONCLUIDA

() OXIMETRO DE PULSO NO PACIENTE E EM

FUNCIONAMENTO

O PACIENTE POSSUI:
ALERGIA CONHECIDA?

(1) SIM QUAIS?

VIA AEREA DIFICIURISCO DE ASPIRAÇAO?

(1) $N A ̈ O$

() SIM EQUIPAMENTOSIASSISTÊNCIA

DISPONIVEIS? () NĀO

RISCO DE PERD
CRIANCAS) ?

CRIANÇA
( ) NÄO
( ) $S I M$

URAC ÃO PREVISTA DA CIRURG

DURAÇÃO PREVISTA DA CIRURG

UAIS?

ANESTESIA

ALGUMA PREOCUPAÇÃO ESPECÍFICA EM RELAÇÃO A ESSE PACIENTE?

\section{ENFERMAGEM}

() MATERIAIS(INSTRUMENTAIS, PRÓTESES) PRESENTES

E DENTRO DO PRAZO DE ESTERILIZAÇĀO?

QUESTŐES RELACIONADAS A EQUIPAMENTOS?

OUTRAS PREOCUPAÇÕES?

EXAMM
() SIM
PROF

SIMS DE IMAGEMM ESTAOO DISPONIVEIS?

SIM () NẢO SE APLICA

OBIANA FOI REALIZADA NOS

\begin{tabular}{cc|c} 
ÚLTIMOS 60 MINUTOS? & () SIM \\
$\begin{array}{ccc}\text { () NÃO SE APLICA } & \text { () SIM HORA:- } & \text { () OUT }\end{array}$
\end{tabular}

QUAL? $\begin{array}{ll}\text { ACESSO } & \text { ( ) SIM ( )NÃO } \\ \text { ENDOVENOSO () AINDA NÄO }\end{array}$ 